\title{
Heart of Darkness: The Downside of Trastuzumab
}

\section{Citation}

Hayes, D. F., and M. H. Picard. 2006. "Heart of Darkness: The Downside of Trastuzumab."

Journal of Clinical Oncology 24 (25) (August 14): 4056-4058. doi:10.1200/jco.2006.07.5143.

\section{Published Version}

doi:10.1200/jco.2006.07.5143

\section{Permanent link}

http://nrs.harvard.edu/urn-3:HUL.InstRepos:29666955

\section{Terms of Use}

This article was downloaded from Harvard University's DASH repository, and is made available under the terms and conditions applicable to Other Posted Material, as set forth at http:// nrs.harvard.edu/urn-3:HUL.InstRepos:dash.current.terms-of-use\#LAA

\section{Share Your Story}

The Harvard community has made this article openly available.

Please share how this access benefits you. Submit a story.

Accessibility 


\title{
Heart of Darkness: The Downside of Trastuzumab
}

\author{
Daniel F. Hayes, Breast Oncology Program, University of Michigan Comprehensive Cancer Center, Ann Arbor, MI \\ Michael H. Picard, Cardiac Ultrasound Laboratory, Cardiology Division, Massachusetts General Hospital and Harvard Medical \\ School, Boston, MA
}

Trastuzumab has been shown to be quite effective in reducing suffering and mortality from breast cancer in both the metastatic ${ }^{1}$ and adjuvant settings. ${ }^{2-5}$ With short follow-up, remarkably consistent results across five adjuvant, prospective, randomized clinical trials suggest that trastuzumab may decrease the odds of distant recurrence and mortality by approximately one half and one third, respectively. Dr George Sledge, who discussed the first presentations of the adjuvant trials at the 2005 Annual Meeting of the American Society of Clinical Oncology, proclaimed these results “astonishing," and we agree (oral communication, May 2005).

However, there is a dark side to trastuzumab that may limit its utility in some patients. Although in general, trastuzumab has been extremely well tolerated, a surprisingly high incidence of congestive heart failure (CHF) was observed in the early studies of metastatic disease, especially in patients who were treated concurrently with doxorubicin. ${ }^{1,6}$ With this knowledge, most subsequent trial designs, particularly those in the adjuvant setting, avoided concurrent anthracycline and trastuzumab therapy, and included careful baseline and serial monitoring of cardiac function. Early results from these trials suggest that approximately $5 \%$ of all patients treated with adjuvant trastuzumab, either with nonanthracycline chemotherapy such as the taxanes or vinorelbine, or after all chemotherapy is complete, will develop some objective evidence of systolic cardiac dysfunction. ${ }^{2,4,5,7}$ Approximately $1 \%$ of patients will develop symptomatic $\mathrm{CHF}^{3,8,9}$ These rates are approximately four to five times higher than in control patients who did not receive trastuzumab, and the absolute difference in echocardiogram or scintigram-detected cardiac dysfunction between trastuzumab-treated and control patients seems to be approximately $3 \%$ to $4 \%$.

These observations raise several questions. First, given the enormous benefit of trastuzumab, is pre-existing cardiac dysfunction, especially if it is asymptomatic, sufficient reason to withhold the drug? Are the classic risk factors for cardiac disease, such as hypertension, diabetes, and family history, important predictors of trastuzumab-induced CHF? Is trastuzumab-related CHF reversible if the agent is discontinued, and is it safe to reinitiate this potentially life-saving agent if a patient has developed cardiac dysfunction while receiving it previously? Are there ways to avoid or minimize trastuzumab-related cardiac dysfunction? Will the incidence of this complication increase with longer follow-up of women who receive adjuvant trastuzumab? Finally, what is the mechanism of this perplexing toxicity?

In the last few years, a substantial number of publications have addressed the cardiac toxicity of trastuzumab in both prospective clinical trials and from individual institutional experiences. ${ }^{10,11}$ In this issue of the Journal of Clinical Oncology, Guarneri et al ${ }^{12}$ report their results from a retrospective analysis of 218 metastatic breast cancer patients who received trastuzumab for at least 1 year from 1998 to 2003. In this series, $28 \%$ of patients had some type of cardiac event (CE), and $10.9 \%$ had grade 3 cardiotoxicity, with one cardiac death. Therefore, coupled with the larger, prospective trials, the results from this study provide additional insight into our questions.

Given that all of the clinical trials of trastuzumab have excluded women with obvious pre-existing heart failure, we cannot determine if this condition should preclude a patient's receiving it; however, until data to the contrary are reported, we believe it should. What about those who apparently have healthy hearts but have received pretrastuzumab anthracyclines, which are incorporated into most adjuvant regimens? In the adjuvant, prospective, randomized clinical trials conducted by the National Surgical Adjuvant Breast and Bowel Project (NSABP B-31) and the Breast Cancer Intergroup of North America, $6.7 \%$ of women who had completed four cycles of doxorubicin and cyclophosphamide (AC) were deemed ineligible to proceed to trastuzumab because of left ventricular dysfunction. ${ }^{2}$ We agree that this condition of eligibility was prudent. One might wonder if these patients could have been treated with trastuzumab and monitored closely with discontinuation of the drug on evidence of additional decline in cardiac function. In this regard, a cautionary note comes from the NSABP, which has reported that patients in NSABP B-31 with marginal post-AC left ventricular ejection fractions (LVEF) but who were still eligible for trastuzumab had higher rates of subsequent cardiac dysfunction than those who started trastuzumab with normal cardiac parameters. So for now, the presence of LV dysfunction or heart failure symptoms should exclude the addition of trastuzumab.

The study by Guarneri et $\mathrm{al}^{12}$ may provide additional clues to our questions. Although the study was retrospective and limited by a small number of events, careful analysis of Table 1 suggests that several pre-existing factors may predict trastuzumab CEs. For example, it seems that although only $7 \%$ of women who received radiation to the right side developed a CE, $26 \%$ of those who received radiation to the left side were affected, although these differences did not reach statistical significance. Again considering the limited power, pre-existing diabetes, coronary artery disease, and valvular dysfunction were all associated with apparent higher rates of trastuzumab-related CEs. Curiously, hypertension and older age were not. In the NSABP/Intergroup analysis of adjuvant patients, age and hypertension were risk factors for cardiac dysfunction. ${ }^{2}$ Additional analysis of the NSABP trial data suggest that 
smoking, family history, history of taking hypoglycemic and/or lipid lowering medications, and radiation to the left side were not risk factors. ${ }^{2,9,13}$

The good news, both from Guarneri et al and previously reported studies, is that trastuzumab-related cardiac dysfunction usually seems to be reversible. In this study, 11 (79\%) of 14 women who stopped trastuzumab therapy after developing symptomatic $\mathrm{CHF}$ recovered with appropriate cardiac treatments. Eighty-nine percent of patients with an asymptomatic decrease in LVEF on cardiac imaging recovered, with or without cardiac treatment, after stopping trastuzumab. These data are similar to other studies, and differ substantially from the early experience with doxorubicin-induced cardiac failure, from which few patients recovered. ${ }^{1,9,14-16}$ Remarkably, trastuzumab was not stopped in 17 patients who developed asymptomatic cardiac dysfunction, and 13 of these did well, most without concurrent cardiac treatment. However, such a strategy requires intensive monitoring of cardiac symptoms and function.

This issue raises the secondary question of the definition of heart failure. For example, in the M.D. Anderson (Houston, TX) experience, nearly one third of patients were classified as having a $\mathrm{CE}$, yet a much smaller percentage developed symptoms. Furthermore, four symptomatic patients, including the one patient who died of apparent CHF, had a normal LVEF. These apparent inconsistencies may potentially be explained in several ways. First, the LVEF by echocardiography can be measured by several methods, and each has an associated degree of variability. For this reason, guidelines and standards have been developed to reduce such variability. ${ }^{17}$ Second, although both nuclear and ultrasound methods can measure LVEF accurately, the approaches and the range of normal values differ slightly between these two techniques. Therefore, when one observes an individual patient, it is important to use the same technique throughout the period of monitoring. Third, although the LVEF is a measure used by most clinicians to communicate about systolic ventricular function, it is influenced by both preload (filling pressures) and afterload (blood pressure), two factors that may vary during the course of treatment in these patients. Fourth, and perhaps most important, heart failure must be defined by more than just LVEF. In fact, the common nomenclature known as the New York Heart Association classification is based on symptoms and functional capacity, not the LVEF. Some patients can remain compensated or asymptomatic despite a significant decrease in LVEF-in some of these patients, the symptoms can be unmasked with more rigorous activity or exercise. Symptoms can also occur in the absence of a decrease in LVEF (the syndrome of diastolic heart failure). This isolated diastolic heart failure is found in up to one third of patients with symptomatic heart failure, and its treatment differs from that of those with systolic heart failure.

Heart failure symptoms are often nonspecific and may mimic those of pulmonary disease, so thoughtful integration of cardiac examination and ventricular imaging are critical. Careful and frequent monitoring of patients treated with trastuzumab should include history and physical examination in addition to noninvasive imaging of ventricular function. In summary, until additional evidence is accumulated from the randomized trials, we should err on the side of caution in the use of this agent when either clinical or echocardiographic/scintigraphic evidence of heart failure exists or develops. New methods for early noninvasive detection of cardiac dysfunction are under development and validation. These methods, including tissue Doppler echocardiography, may enable more sensitive detection of subtle changes in myocardial function before those detected by measurement of LVEF, but careful studies will be necessary to ensure that we do not withhold trastuzumab on the basis of a surrogate indication of cardiac dysfunction. ${ }^{18}$

In addition to avoiding trastuzumab in women with cardiac problems, several strategies have been adopted or proposed to minimize cardiac toxicity. After the initial observations, most investigators quickly eliminated concurrent doxorubicin and trastuzumab, and general guidelines state that the combination is contraindicated. ${ }^{1,19}$ However, in a few preliminary trials, trastuzumab has been given concurrently with less cardiotoxic anthracycline compounds, such as epirubicin or liposomal-encapsulated doxorubicin, and the results have been promising. ${ }^{20-22}$ Another strategy has been to shorten the duration of adjuvant trastuzumab therapy. In a prospective randomized trial conducted in Finland (HERFin), with short follow-up and few events, 12 weeks of trastuzumab given with post-AC docetaxel or vinorelbine seems to be as effective and perhaps safer than longer therapy. ${ }^{4}$ Investigators in the HERceptin Adjuvant trial chose to delay initiation of trastuzumab until after all chemotherapy was complete. However, incidence of cardiac events seems similar to that in other adjuvant trials of 12 months of trastuzumab. ${ }^{3}$ Although intriguing, these strategies must be considered investigational, and they are not recommended for routine clinical care.

In the M.D. Anderson experience, prior treatment with doxorubicin, surprisingly, was not a risk factor for CE. Nonetheless, another strategy to avoid CHF might be to eliminate anthracyclines altogether in the adjuvant setting. Because preclinical studies had suggested possible synergy between trastuzumab and two nonanthracycline chemotherapeutic agents (docetaxel and platinum salts), a prospective adjuvant trial conducted by the Breast Cancer International Research Group contained a third arm in which no anthracycline was given. In this arm, patients received docetaxel, carboplatin, and trastuzumab. ${ }^{5}$ As expected, cardiac events in the docetaxel, carboplatin, and trastuzumab arm were similar to those in the control group that received only AC followed by docetaxel but no trastuzumab. However, although not statistically significant, the reduction in breast cancer recurrences in this third arm did not seem to be as robust, relative to control, as in the group that received AC-docetaxel plus trastuzumab.

Another possibility is that cardiac toxicity might be related to trastuzumab itself, and therefore it might not occur with other agents that target HER-2 in different ways. Recently reported results suggest that the pan-epidermal growth factor receptor family tyrosine kinase inhibitor, lapatinib, seems active against HER-2positive breast cancer, and that this agent might have less cardiac toxicity than has been observed with trastuzumab. ${ }^{23,24}$ More mature follow-up is needed to determine if the efficacy and safety profiles are similar to those of trastuzumab.

Finally, what is the mechanism of trastuzumab-related cardiac dysfunction? Several preclinical models have suggested that the epidermal growth factor receptor family is an important component of cardiac development; in adults, the HER-2 receptor seems to participate in regulation of growth, function, repair, and survival of cardiomyocytes. Addition studies suggest that trastuzumab has a direct action on human cardiomyocytes. ${ }^{25-28}$ Therefore, the 
combination of induction of cardiac injury with preceding doxorubicin followed by inhibition of cardiac repair mediated by erbB2 may result in the cardiac dysfunction associated with trastuzumab.

In summary, the stakes are high. It is essential that we continue to deliver the "astonishing" beneficial effects of trastuzumab therapy to those who will benefit, while avoiding major short- and long-term toxicities. These challenges exist in the context of tightening health care dollars, both in the United States and abroad. We eagerly await release of analyses of the large ongoing and future randomized trials that will permit additional individualization of this exciting approach.

\section{(C) 2006 by American Society of Clinical Oncology \\ REFERENCES}

1. Slamon DJ, Leyland-Jones B, Shak S, et al: Use of chemotherapy plus a monoclonal antibody against HER2 for metastatic breast cancer that overexpresses HER2. N Engl J Med 344:783-792, 2001

2. Romond EH, Perez EA, Bryant J, et al: Trastuzumab plus adjuvant chemotherapy for operable HER2-positive breast cancer. N Engl J Med 353:1673-1684, 2005

3. Piccart-Gebhart MJ, Procter M, Leyland-Jones B, et al: Trastuzumab after adjuvant chemotherapy in HER2-positive breast cancer. N Engl J Med 353:16591672,2005

4. Joensuu $\mathrm{H}$, Kellokumpu-Lehtinen $\mathrm{PL}$, Bono $\mathrm{P}$, et al: Adjuvant docetaxel or vinorelbine with or without trastuzumab for breast cancer. $N$ Engl J Med 354:809-820, 2006

5. Slamon D, Eiermann W, Robert $N$, et al: Phase III randomized trial comparing doxorubicin and cyclophosphamide followed by docetaxel with doxorubicin and cyclophosphamide followed by docetaxel and trastuzumab with docetaxel, carboplatin and trastuzumab in HER-2 positive early breast cancer patients: BCIRG 006 study. Breast Cancer Res Treat 94:S5, 2005 (abstr 1)

6. Ewer MS, Gibbs HR, Swafford J, et al: Cardiotoxicity in patients receiving trastuzumab (Herceptin): Primary toxicity, synergistic or sequential stress, or surveillance artifact? Semin Oncol 26:96-101, 1999

7. Piccart-Gebhart MJ, Procter $M$, Leyland-Jones $B$, et al: Trastuzumab after adjuvant chemotherapy in HER2-positive breast cancer. N Engl J Med 353:16591672, 2005

8. Perez EA, Rodeheffer R: Clinical cardiac tolerability of trastuzumab. J Clin Oncol 22:322-329, 2004

9. Tan-Chiu E, Yothers G, Romond E, et al: Assessment of cardiac dysfunction in a randomized trial comparing doxorubicin and cyclophosphamide followed by paclitaxel, with or without trastuzumab as adjuvant therapy in node-positive, human epidermal growth factor receptor 2-overexpressing breast cancer: NSABP B-31. J Clin Oncol 23:7811-7819, 2005

10. Baselga J, Tripathy D, Mendelsohn J, et al: Phase II study of weekly intravenous recombinant humanized anti-p185HER2 monoclonal antibody in patients with HER2/neu-overexpressing metastatic breast cancer. J Clin Oncol 14:737-744, 1996

11. Pegram M, Lipton A, Hayes DF, et al: Phase II study of receptor-enhanced chemosensitivity using recombinant humanized anti-p185HER2/neu monoclonal antibody plus cisplatin in patients with HER2/neu-overexpressing metastatic breast cancer refractory to chemotherapy treatment. J Clin Oncol 16:2659-2671, 1998

12. Guarneri V, Lenihan DJ, Valero V, et al: Long-term cardiac tolerability of trastuzumab in metastatic breast cancer: The M.D. Anderson Cancer Center experience. J Clin Oncol 24:4107-4115, 2006

13. Geyer CE Jr, Bryant J, Yothers G, et al: Update of cardiac dysfunction on NSABP B-31:A randomized trial of sequential AC->paclitaxel vs. AC->paclitaxel with trastuzumab. J Clin Oncol 24:23s, 2006 (suppl; abstr 581)

14. Ewer MS, Vooletich MT, Durand JB, et al: Reversibility of trastuzumabrelated cardiotoxicity: New insights based on clinical course and response to medical treatment. J Clin Oncol 23:7820-7826, 2005

15. von Hoff DD, Layard MW, Basa $P$, et al: Risk factors for doxorubicininduced congestive heart failure. Ann Intern Med 91:710-717, 1979

16. Doroshow JH: Doxorubicin-induced cardiac toxicity. N Engl J Med 324: 843-845, 1991

17. Lang RM, Bierig M, Devereux RB, et al: Recommendations for chamber quantification: A report from the American Society of Echocardiography's Guidelines and Standards Committee and the Chamber Quantification Writing Group, developed in conjunction with the European Association of Echocardiography, a branch of the European Society of Cardiology. J Am Soc Echocardiogr 18:14401463,2005

18. Neilan TG, Jassal DS, Perez-Sanz TM, et al: Tissue Doppler imaging predicts left ventricular dysfunction and mortality in a murine model of cardiac injury. Eur Heart J 10.1093/eurheartj/ehl013 [epub ahead of print on May 22, 2006]

19. Ellis $M$, Hayes DF, Lippman ME: Treatment of metastatic breast cancer, in Harris J, Lippman M, Morrow M, et al (eds): Diseases of the Breast (ed 3). Philadelphia, PA, Lippincott Williams \& Wilkins, 2004, pp 1101-1162

20. Buzdar AU, Ibrahim NK, Francis $D$, et al: Significantly higher pathologic complete remission rate after neoadjuvant therapy with trastuzumab, paclitaxel, and epirubicin chemotherapy: Results of a randomized trial in human epidermal growth factor receptor 2-positive operable breast cancer. J Clin Oncol 23:36763685, 2005

21. Untch M, HimsI I, Kahlert S, et al: Anthracycline and trastuzumab in breast cancer treatment. Oncology (Williston Park) 18:59-64, 2004

22. Chia S, Clemons M, Martin LA, et al: Pegylated liposomal doxorubicin and trastuzumab in HER-2 overexpressing metastatic breast cancer: A multicenter phase II trial. J Clin Oncol 24:2773-2778, 2006

23. Geyer CE Jr, Cameron D, Lindquist D, et al: A phase III randomized, open-label, international study comparing lapatinib and capecitabine vs. capecitabine in women with refractory advanced or metastatic breast cancer (EGF100151). Presented at the 42nd Annual Meeting of the American Society of Clinical Oncology, Orlando, FL, June 2-6, 2006

24. Perez EA, Byrne J: Results of an analysis of cardiac function in 2,812 patients treated with lapatinib. J Clin Oncol 24:23s, 2006 (suppl; abstr 583)

25. Schneider JW, Chang AY, Rocco TP: Cardiotoxicity in signal transduction therapeutics: ErbB2 antibodies and the heart. Semin Oncol 28:18-26, 2001

26. Behr TM, Behe M, Wormann B: Trastuzumab and breast cancer. N Engl J Med 345:995-996, 2001

27. Schneider JW, Chang AY, Garratt A: Trastuzumab cardiotoxicity: Speculations regarding pathophysiology and targets for further study. Semin Oncol 29:22-28, 2002

28. Ozcelik C, Erdmann B, Pilz B, et al: Conditional mutation of the ErbB2 (HER2) receptor in cardiomyocytes leads to dilated cardiomyopathy. Proc Natl Acad Sci U S A 99:8880-8885, 2002

\section{Acknowledgment}

Supported by National Institutes of Health Grant No. RO1 CA92461 (D.F.H.) and by the Fashion Footwear Charitable Foundation of New York/QVC Presents Shoes on Sale (D.F.H.).

\section{Authors' Disclosures of Potential Conflicts of Interest}

The authors indicated no potential conflicts of interest.

\section{Author Contributions}

Manuscript writing: Daniel F. Hayes, Michael H. Picard

Final approval of manuscript: Daniel F. Hayes, Michael H. Picard 


\section{Editorials}

Prognostic Markers of Local Relapse in Rectal Cancer: Are We Any Further Forward? Patrick G. Johnston (see article on page 4062)

Thymidine Phosphorylase and Capecitabine: A Predictive Marker for Therapy Selection?

Bert H. O'Neil and Howard L. McLeod (see article on page 4069) 4051

What Is the Right Dose? The Elusive Optimal Biologic Dose in Phase I Clinical Trials Alex A. Adjei (see article on page 4092) 4054 E Heart of Darkness: The Downside of Trastuzumab Daniel F. Hayes and Michael H. Picard (see article on page 4107) 4056 EWhich Breast Cancer Patients Should Really Worry About Radiation-Induced Heart Disease-And How Much?

Abram Recht (see article on page 4100)

\section{Original Reports}

\section{GASTROINTESTINAL CANCER}

\section{(i) Lymph Node Status and TS Gene Expression Are Prognostic Markers in Stage II/III} Rectal Cancer After Neoadjuvant Fluorouracil-Based Chemoradiotherapy

Torsten Liersch, Claus Langer, B. Michael Ghadimi, Bettina Kulle, Daniela E. Aust, Gustavo B. Baretton, Wolfgang Schwabe, Peter Häusler, Heinz Becker, and Christiane Jakob (see editorial on page 4049)

(i) Thymidine Phosphorylase Expression Is Associated With Response to Capecitabine Plus Irinotecan in Patients With Metastatic Colorectal Cancer Neal J. Meropol, Philip J. Gold, Robert B. Diasio, Michael Andria, Mandeep Dhami, Thomas Godfrey, Albert J. Kovatich, Kirk A. Lund, Edith Mitchell, and Roland Schwarting (see editorial on page 4051)

Predictors of Recurrence in Patients With T2 and Early T3, NO Adenocarcinoma of the Rectum Treated by Surgery Alone

Aviram Nissan, Alexander Stojadinovic, Jinru Shia, Axel Hoos, Jose G. Guillem, David Klimstra, Alfred M. Cohen, Bruce D. Minsky, Philip B. Paty, and W. Douglas Wong

Pooled Analysis of Safety and Efficacy of Oxaliplatin Plus Fluorouracil/Leucovorin Administered Bimonthly in Elderly Patients With Colorectal Cancer

Richard M. Goldberg, Isabelle Tabah-Fisch, Harry Bleiberg, Aimery de Gramont, Christophe Tournigand, Thierry Andre, Mace L. Rothenberg, Erin Green, and Daniel J. Sargent

Journal of Clinical Oncology (ISSN 0732-183X) is published 36 times a year, three times monthly, by American Society of Clinical Oncology, 1900 Duke St, Suite 200, Alexandria, VA 22314. Periodicals postage is paid at Alexandria, VA, and at additional mailing offices. Publication Mail Agreement Number 863289.

Editorial correspondence should be addressed to Daniel G. Haller, MD, Journal of Clinical Oncology, 330 John Carlyle St, Suite 300, Alexandria, VA 22314. Telephone: (703) 797-1900; Fax: (703) 684-8720. E-mail: jco@asco.org. Internet: www.jco.org.

POSTMASTER: ASCO members send change of address to American Society of Clinical Oncology, 1900 Duke St, Suite 200, Alexandria, VA 22314. Nonmembers send change of address to Journal of Clinical Oncology Customer Service, 330 John Carlyle St, Suite 300, Alexandria, VA 22314.

2007 annual subscription rates, effective September 1, 2006: United States and possessions: individual, $\$ 487$; single issue, $\$ 35$. International: individual, $\$ 678$; single issue, $\$ 45$. Institutions: Tier 1: \$670 US, \$950 Int'l; Tier 2: \$780 US, \$1,060 Int'l; Tier 3: \$1,130 US, \$1,410 Int'l; Tier 4: \$1,245 US; \$1,525 Int'l; Tier 5: contact JCO for a quote. See http://www.jco.org/subscriptions/tieredpricing.shtml for descriptions of each tier. Student and resident: United States and possessions: $\$ 240$; all other countries, $\$ 336$. To receive student/resident rate, orders must be accompanied by name of affiliated institution, date of term, and the signature of program/residency coordinator on institution letterhead. Orders will be billed at individual rate until proof of status is received. Current prices are in effect for back volumes and back issues. Back issues sold in conjunction with a subscription rate are on a prorated basis. Subscriptions are accepted on a 12-month basis. Prices are subject to change without notice. Single issues, both current and back, exist in limited quantities and are offered for sale subject to availability. JCO Legacy Archive (electronic back issues from January 1983 through December 1998) is also available; please inquire. 


\section{PHASE I AND CLINICAL PHARMACOLOGY}

Phase I Dose Escalation and Pharmacokinetic Study of Enzastaurin, an Oral Protein Kinase C Beta Inhibitor, in Patients With Advanced Cancer

Michael A. Carducci, Luna Musib, Merrill S. Kies, Roberto Pili, Mylene Truong, Julie R. Brahmer, Patricia Cole, Rana Sullivan, Jeanne Riddle, Jill Schmidt, Nathan Enas, Vikram Sinha, Donald E. Thornton, and Roy S. Herbst (see editorial on page 4054)

\section{BREAST CANCER}

E (i) Late Cardiac Mortality and Morbidity in Early-Stage Breast Cancer Patients After Breast-Conservation Treatment

Eleanor E.R. Harris, Candace Correa, Wei-Ting Hwang, Jessica Liao, Harold I. Litt, Victor A. Ferrari, and Lawrence J. Solin (see editorial on page 4059)

E Long-Term Cardiac Tolerability of Trastuzumab in Metastatic Breast Cancer: The M.D. Anderson Cancer Center Experience

Valentina Guarneri, Daniel J. Lenihan, Vicente Valero, Jean-Bernard Durand, Kristine Broglio, Kenneth R. Hess, Laura Boehnke Michaud, Ana M. Gonzalez-Angulo, Gabriel N. Hortobagyi, and Francisco J. Esteva (see editorial on page 4056)

\section{COST EFFECTIVENESS}

Cost-Effectiveness Analysis of Computerized Tomography in the Routine Follow-Up of Patients After Primary Treatment for Hodgkin's Disease

Beverly A. Guadagnolo, Rinaa S. Punglia, Karen M. Kuntz, Peter M. Mauch, and Andrea K. Ng

\section{AIDS-RELATED CANCER}

\section{를 (1) Phase II Trial of CHOP Plus Rituximab in Patients With HIV-Associated} Non-Hodgkin's Lymphoma

François Boué, Jean Gabarre, Christian Gisselbrecht, Jacques Reynes, Antoine Cheret, Fabrice Bonnet, Eric Billaud, Martine Raphael, Remi Lancar, and Dominique Costagliola

\section{SUPPORTIVE CARE AND QUALITY OF LIFE}

\section{Outpatient Oral Antibiotics for Febrile Neutropenic Cancer Patients Using a Score} Predictive for Complications

Jean Klastersky, Marianne Paesmans, Aspasia Georgala, Frédérique Muanza, Barbara Plehiers, Laurent Dubreucq, Yassine Lalami, Michel Aoun, and Martine Barette

\section{HEMATOLOGIC MALIGNANCIES}

Prognostic Impact of Germinal Center-Associated Proteins and Chromosomal Breakpoints in Poor-Risk Diffuse Large B-Cell Lymphoma

Gustaaf W. van Imhoff, Evert-Jan G. Boerma, Bronno van der Holt, Ed Schuuring, Leo F. Verdonck, Hanneke C. Kluin-Nelemans, and Philip M. Kluin

E Phase II Trial of CHOP Chemotherapy Followed by Tositumomab/lodine I-131 Tositumomab for Previously Untreated Follicular Non-Hodgkin's Lymphoma: Five-Year Follow-Up of Southwest Oncology Group Protocol S9911

Oliver W. Press, Joseph M. Unger, Rita M. Braziel, David G. Maloney, Thomas P. Miller, Michael LeBlanc, and Richard I. Fisher

\section{BONE MARROW TRANSPLANTATION}

\section{Eactors Associated With Outcomes in Allogeneic Hematopoietic Cell Transplantation} With Nonmyeloablative Conditioning After Failed Myeloablative Hematopoietic Cell Transplantation

Frédéric Baron, Rainer Storb, Barry E. Storer, Michael B. Maris, Dietger Niederwieser, Judith A. Shizuru, Thomas R. Chauncey, Benedetto Bruno, Stephen J. Forman, Peter A. McSweeney, Richard T. Maziarz, Michael A. Pulsipher, Edward D. Agura, James Wade, Mohamed Sorror, David G. Maloney, and Brenda M. Sandmaier 
(i) Impact of a Multi-Disciplinary Patient Education Session on Accrual to a Difficult Clinical Trial: The Toronto Experience With the Surgical Prostatectomy Versus Interstitial Radiation Intervention Trial

Kris Wallace, Neil Fleshner, Michael Jewett, Joan Basiuk, and Juanita Crook

HEAD AND NECK CANCER

Phase I/II Study of Docetaxel, Cisplatin, and Concomitant Boost Radiation for Locally Advanced Squamous Cell Cancer of the Head and Neck

Anne S. Tsao, Adam S. Garden, Merrill S. Kies, William Morrison, Lei Feng, J. Jack Lee, Fadlo Khuri, Ralph Zinner, Jeffery Myers, Vassiliki Papadimitrakopoulou, Jan Lewin, Gary L. Clayman, K. Kian Ang, and Bonnie S. Glisson

Increased Epidermal Growth Factor Receptor Gene Copy Number Is Associated With Poor Prognosis in Head and Neck Squamous Cell Carcinomas

Christine H. Chung, Kim Ely, Loris McGavran, Marileila Varella-Garcia, Joel Parker, Natalie Parker, Carolyn Jarrett, Jesse Carter, Barbara A. Murphy, James Netterville, Brian B. Burkey, Robert Sinard, Anthony Cmelak, Shawn Levy, Wendell G. Yarbrough, Robbert J.C. Slebos, and Fred R. Hirsch

Disadvantage of Men Living Alone Participating in Radiation Therapy Oncology Group Head and Neck Trials

Andre A. Konski, Thomas F. Pajak, Benjamin Movsas, James Coyne, Jonathan Harris, Clement Gwede, Adam Garden, Sharon Spencer, Christopher Jones, and Deborah Watkins-Bruner

\section{GENITOURINARY CANCER}

Risk of Cardiovascular Mortality in Prostate Cancer Patients in the Rotterdam Randomized Screening Trial

Suzie J. Otto, Fritz H. Schröder, and Harry J. de Koning 4184

Six-Month Androgen Suppression Plus Radiation Therapy Compared With Radiation Therapy Alone for Men With Prostate Cancer and a Rapidly Increasing Pretreatment Prostate-Specific Antigen Level

Anthony V. D'Amico, Marian Loffredo, Andrew A. Renshaw, Brittany Loffredo, and Ming-Hui Chen 4190

\section{MELANOMA}

Randomized Multicenter Trial of Hyperthermic Isolated Limb Perfusion With Melphalan Alone Compared With Melphalan Plus Tumor Necrosis Factor: American College of Surgeons Oncology Group Trial Z0020

Wendy R. Cornett, Linda M. McCall, Rebecca P. Petersen, Merrick I. Ross, Henry A. Briele, R. Dirk Noyes, Jeffrey J. Sussman, William G. Kraybill, John M. Kane III, H. Richard Alexander, Jeffrey E. Lee,

Paul F. Mansfield, James F. Pingpank, David J. Winchester, Richard L. White Jr, Vijaya Chadaram,

James E. Herndon II, Douglas L. Fraker, and Douglas S. Tyler

\section{PEDIATRIC ONCOLOGY}

Phase III Study of Craniospinal Radiation Therapy Followed by Adjuvant Chemotherapy for Newly Diagnosed Average-Risk Medulloblastoma

Roger J. Packer, Amar Gajjar, Gilbert Vezina, Lucy Rorke-Adams, Peter C. Burger, Patricia L. Robertson, Lisa Bayer, Deborah LaFond, Bernadine R. Donahue, MaryAnne H. Marymont, Karin Muraszko,

James Langston, and Richard Sposto

\section{Art of Oncology}

Discussing Prognosis: “How Much Do You Want to Know?" Talking to Patients Who Are Prepared for Explicit Information

Anthony L. Back and Robert M. Arnold (see article on page 4214)

Discussing Prognosis: “How Much Do You Want to Know?" Talking to Patients Who Do Not Want Information or Who Are Ambivalent

Anthony L. Back and Robert M. Arnold (see article on page 4209) 


\section{Correspondence}

\section{Immunotherapy and Prognostic Factors}

Luca A. Fumagalli and Fernando Brivio

\section{In Reply}

Frede Donskov and Hans von der Maase

\section{Fertility Preservation Strategies for Breast Cancer Patients}

Lucia Del Mastro and Marco Venturini

In Reply

Kutluk H. Oktay and Stephanie J. Lee

\section{Sedation and Expertise in Palliative Care}

José António Ferraz Gonçalves

\section{Epoetin Versus Darbepoetin Conundrum Compromise}

Archie Bleyer

Errata

4223

\section{Also in This Issue}

Announcements

Information for Contributors

Current Abstracts

Calendar of Oncology Events

(i) Online supplementary information available at www.jco.org

Article was published online ahead of print at www.jco.org

Additional correspondence available online at www.jco.org 